\title{
Orden social y conflicto en la teoría de los sistemas de Niklas Luhmann
}

\author{
Conflict and social order in Niklas Luhmann's systems theory \\ Juan Pablo Gonnet (jpgonnet@conicet.gov.ar) Centro de Estudios e Investigaciones sobre Cultura y \\ Sociedad, Universidad Nacional de Córdoba (Córdoba, Argentina) ORCID: 0000-0002-1851-9383
}

\begin{abstract}
Through the assimilation of contingency and complexity, Niklas Luhmann's social systems theory proposes a conceptualization of social order that is sensitive to those phenomena that challenge established institutional logics. This opening allows the integration of variation, change and conflict as possibilities always open in the reproduction of social systems as phenomena that are not antagonistic to social order. The purpose of this article is to analyse, specifically, the treatment of conflict that emerges from this conceptual manoeuvre. Our hypothesis is that, although the conflict is formally integrated in this theory, it is weakly explained as it partially recognizes the social conditions that make it viable. We argue that the probable cause of this deficit lies in the reproduction of a regulative conception of social order that is not sufficiently discussed regarding contingency and complexity.
\end{abstract}

Keywords: conflict, double contingency, social structures, social order, social system.

\section{Resumen}

A través de la asimilación de la contingencia y la complejidad, la teoría general de los sistemas sociales de Niklas Luhmann propone una conceptualización del orden social que se muestra sensible a aquellos fenómenos que desafían a las lógicas institucionales establecidas. Dicha apertura ha permitido integrar teóricamente a la variación, al cambio y al conflicto como posibilidades siempre abiertas en la reproducción de los sistemas sociales. Es decir, como fenómenos no antagónicos con el orden social. El objetivo de este artículo es analizar, específicamente, el tratamiento del conflicto que se desprende de esta maniobra conceptual. Nuestra hipótesis es que, si bien el conflicto se encuentra formalmente integrado en esta teoría del orden, el mismo es explicado de un modo deficiente puesto que solo pueden reconocerse parcialmente aquellas condiciones sociales que lo viabilizan. Sostenemos que la causa probable de este déficit se halla en la reproducción de una concepción regulativa de orden social que no se encuentra suficientemente problematizada en relación con los supuestos de contingencia y complejidad.

Palabras clave: conflicto, doble contingencia, estructuras sociales, orden social, sistema social.

\section{Introducción}

Frecuentemente en la historia del pensamiento sociológico se ha entendido al conflicto como un fenómeno contrario y hasta antagónico con respecto al orden social, especialmente, cuando este último es observado como un equivalente al consenso. Dicha situación ha propiciado un acercamiento 
diferenciado a ambos fenómenos dificultando un tratamiento unificado. Aquello que resulta útil para explicar el orden no lo es para el caso del conflicto y viceversa. La teoría de los sistemas sociales de Niklas Luhmann busca trascender este abordaje dualista proponiendo una imagen de orden social que resulta compatible con el conflicto. Dicha alternativa teórica encuentra sus fundamentos en los presupuestos de complejidad y contingencia de los que parte esta perspectiva sistémica. Todo sistema social opera en entornos que poseen más complejidad de la que puede abarcar, por lo que debe seleccionar estrategias de reducción de complejidad que, inevitablemente, se presentan como contingentes. De esta manera, todos aquellos acontecimientos que impliquen algún tipo de desviación o desafío con respecto a dichas selecciones son contemplados como alternativas siempre disponibles y, por tanto, plenas de sentido para los sistemas sociales. Por esta razón, el conflicto no puede concebirse como un acontecimiento contradictorio u obturado por el orden social. El mismo aparece como una alternativa siempre disponible en el proceso de su producción y reproducción, sin que esto propicie su disolución.

En el siguiente trabajo analizamos críticamente el tratamiento del conflicto que se desprende de esta maniobra conceptual. Básicamente, observamos que la compatibilidad planteada entre orden y conflicto es parcial en tanto éste último no puede ser explicado por el primero. Así, no es el orden social el que viabiliza al conflicto, sino que es la complejidad y la contingencia propia de los sistemas sociales la que lo hace. El conflicto aparece como aquello que escapa al orden, aunque pueda ser asimilado por éste. La hipótesis que deseamos desarrollar sostiene que, si bien el conflicto es formalmente integrado en la teoría de los sistemas sociales de Luhmann, el conflicto se haya deficientemente explicado al no poder ser entendido más que como una alternativa cuya ocurrencia es siempre posible y, en el mejor de los casos, más o menos probable.

Adelantando un poco nuestro argumento, consideramos que las razones de este déficit se hayan en una concepción de orden social que se encuentra asociada al problema del control. La particularidad de la teoría de los sistemas a la que suscribe Luhmann no subordina el control a la eliminación de la contingencia. De hecho, se entiende que el control no requiere necesariamente de la reproducción de las estructuras sistémicas. Si éstas controlasen todos los sucesos del sistema, entonces no habría lugar para el conflicto (al menos sin que éste amenazase la supervivencia del sistema). Sin embargo, esto no implica que las estructuras no tengan importancia. De hecho, resultan instructivas para el reconocimiento de desviaciones, variaciones, decepciones y perturbaciones, permitiendo incorporarlas al sistema como informaciones útiles para la continuación de las operaciones. Ahora bien, más allá de reconocer a estos acontecimientos como constitutivos de los procesos sistémicos, surge la pregunta acerca de las razones de su ocurrencia. Desde una teoría que entiende al orden social como control, solo pueden aparecer como hechos generados por la incertidumbre e inestabilidad (complejidad) en la que operan los sistemas y por el carácter contingente de sus estructuras. Stephen Fuchs plantea acertadamente que para Luhmann: "EI orden social no radica en la exclusión del malentendido, del conflicto, de la desviación o de la decepción, descansa más bien en los sistemas comunicativos que deciden cómo manejar la desviación, el conflicto y el malentendido" (Fuchs 2000:209).

En conexión con esto, el conflicto tiende a aparecer como algo que escapa al control de las estructuras o, mejor dicho, que exhibe sus límites. Gracias a ello, configura una "señal de alarma" para el sistema, el cual puede detectar la pérdida de la capacidad coordinadora de las estructuras. En este sentido, a pesar de que efectivamente se entiende que el conflicto no resulta antagónico con el orden, es claro que tampoco puede ser acabadamente explicado por éste. El mismo es habilitado por la contingencia de la estructura, pero no explicado por ella. Debido a esto, consideramos que la visión del orden como control termina constituyéndose en un obstáculo epistemológico para una integración acabada del conflicto en la teoría 
del orden social. El conflicto tiene que presentarse, inevitablemente, como un acontecimiento residual, o sea como un hecho que es reconocido por la teoría, pero cuyo origen resulta incierto.

En este espacio pretendemos desarrollar este problema a partir del análisis de la conceptualización del conflicto que Luhmann elabora, principalmente, en el libro Sistemas Sociales, en donde se encuentra más acabadamente formulada su teoría general. No obstante, también recuperamos otros textos del autor en donde se retoma directa o indirectamente la temática. Presentamos nuestro argumento en cinco momentos. En primer lugar, reconstruimos las dimensiones básicas de la teoría del orden social propuesta por Luhmann en conexión con los desarrollos de la teoría de los sistemas. En segundo lugar, analizamos el modo en que el conflicto es integrado en esta teoría del orden. Luego, en tercer lugar, atendemos a los procesos sociales que desencadenan los conflictos; entre ellos, la construcción de un tipo de sistema (orden) social particular. En cuarto lugar, describimos los mecanismos que, para Luhmann, viabilizan la reproducción y, en algunas ocasiones, la institucionalización de los conflictos en la sociedad. En todos estos niveles de la teorización luhmanniana buscamos justificar nuestra hipótesis interpretativa. Finalmente, a modo de cierre, en el último apartado, proponemos algunos desafíos que deberá enfrentar una teoría del orden social que desee reconocer la contingencia del conflicto y que, a la vez, pretenda no descuidar su capacidad explicativa e interpretativa.

\section{El orden social como sistema}

Desde sus orígenes la sociología se preocupó por la cuestión del orden social, es decir, por el modo en que se coordinan los comportamientos individuales dando lugar a la emergencia de fenómenos colectivos. A pesar de esto, Luhmann destaca que no fue hasta la aparición de la teoría sociológica de Parsons que se vislumbró a la temática como un problema y no como un hecho autoevidente de la vida con otros. Invocando al teorema de la doble contingencia (de aquí en adelante DC), Parsons sociologizó el problema hobbesiano del orden y le otorgó una nueva solución. La DC describe una situación de interacción en la cual las acciones de los interlocutores (ego y alter) son contingentes con respecto a las acciones del otro. Por un lado, la acción de ego depende de la reacción de alter y, a su vez, la acción de alter depende de la acción que desarrolle ego. Sin solución a esta situación de DC no solo no es factible el orden, sino que tampoco es posible la acción. La alternativa sugerida por Parsons se inscribe en el campo de una teoría general de la acción. Es en ésta en donde encontramos la respuesta al problema del orden. Para Parsons, la acción configura un sistema que integra un orden normativo de expectativas a partir del cual es posible la orientación coordinada de la acción en una situación.

Para Luhmann resulta productivo atender al problema del orden social por medio de la DC. A pesar de esto, discrepa con el tratamiento que le otorga el sociólogo norteamericano. De acuerdo con Luhmann, la DC no puede ser entendida meramente como un problema analítico para la sociología, más bien debe abordarse como un problema mundano, concreto y permanente con el que deben lidiar los seres humanos y los sistemas sociales. En este sentido, la DC opera como una fuente de estímulos permanente para la construcción y/o transformación del orden. En esta dirección, se entiende que el orden social es posible "gracias a" y no "en contra de" la DC. La posibilidad de reinscribir el problema en el mismo funcionamiento de los sistemas sociales le otorga a la teoría mayor dinamismo y apertura hacia la consideración de aquello que escapa a una solución particular y circunstancial de la DC.

Por otra parte, Luhmann desacuerda con el modo en que Parsons conceptualiza a la contingencia en el marco de la DC. Para este último, la contingencia indicaba dependencia con respecto a las acciones del otro. Así, la DC describía una situación interactiva de mutua dependencia. Concebida de esta manera, la 
solución al problema solo podía remontarse a una circunstancia externa y previa al círculo contingente. Ninguna acción es posible si ego hace depender su comportamiento del de alter, por lo que la acción debe depender de un orden previamente existente y definido. Para Luhmann, en cambio, la contingencia remite a aquello que no es necesario ni imposible, es decir, a lo que "es" (Luhmann 1998:115), pero que también puede ser de otro modo. En el marco de la DC esto implica que la ocurrencia de la acción social no es dependiente de la acción de otro, aunque inevitablemente se relacione y se referencie en ella. La DC describe una contingencia estrictamente social; todo lo que sucede en su ámbito pertenece al orden social y su carácter contingente resulta independizado de las intenciones y las motivaciones de los actores. A partir de esto, Luhmann postula que los sistemas son sistemas autónomos con respecto a los seres humanos, quienes se hayan ubicados en el entorno de éstos, ya sea como sistemas psíquicos o vivos. Así es posible comprender que el orden social no requiere de la eliminación de las diferencias individuales. Lo social y la autonomía individual no resultan incompatibles. Esta decisión desacredita aquella imagen que supone que el orden requiere de la eliminación de las conductas adversas.

Por el lado de la solución de la DC, ambos autores comparten que resulta insuficiente apelar a hipótesis en torno a acuerdos o negociaciones interactivas. Tampoco el proseguir intereses individuales se vislumbra como adecuado. Sin embargo, Luhmann no acuerda con buscar la solución en una teoría general de la acción que vía socialización integre un componente normativo orientativo. Asumir esto, llevaría a la negación de la DC. Adicionalmente, Luhmann discrepa en el excesivo énfasis que pone Parsons en las estructuras culturales, valorativas y normativas como las únicas capaces de resolver la DC. En el mejor de los casos, esto constituye solo una de las posibilidades para la producción del orden entre otras. Por ejemplo, existen equivalentes funcionales como los medios de comunicación simbólicamente generalizados (poder, dinero, amor, verdad, entre otros), cuya constitución es antagónica con las formas de reducción de complejidad prefiguradas por los sustratos culturales que resuelven el problema del orden social temporalmente en el pasado, objetivamente en los contenidos compartidos y socialmente en la identidad de los interlocutores. Este reduccionismo sería un obstáculo no solo para la comprensión del orden social, sino también para la inclusión de los conflictos como formas de orden.

A partir de estas apropiaciones críticas de la teoría parsoniana, Luhmann define su tentativa de respuesta a la pregunta por el cómo es posible el orden social. Luhmann desea mostrar que es la misma situación de improbabilidad la que dinamiza la emergencia de los sistemas sociales: "bajo esta condición de la DC, cualquier autodeterminación surgida arbitrariamente y calculada de cualquier manera, ganará valor de información y de relación para otras actuaciones" (Luhmann 1998:124). El círculo doblecontigente se rompe cuando sucede algo, cuando se observa una selección y se actúa en consecuencia. Cualquier casualidad surgida en el ámbito de la DC permite lidiar con ella, es decir, establecerse como una estructura condicionante para las partes: "El orden social se lleva a cabo cuando alguien hace una propuesta o efectúa una acción que pone a los otros en una situación de reacción: aceptar o rechazar la propuesta" (Luhmann 2002:329). La contingencia social no desaparece, pero toda instancia siguiente se articulará positiva o negativamente con base a la determinación definida. De este modo, la solución a la DC es provisoria y momentáneamente satisfactoria, susceptible de ser defraudada por nuevos acontecimientos. Como mencionamos al principio, el orden precisa de estructuras, pero no de su estabilidad. Las estructuras, que para Luhmann se materializan en expectativas, no eliminan la indeterminabilidad, pero la reducen a dos opciones: la conformidad o la decepción. El orden social es un sistema que no desarrolla preferencia alguna por estas alternativas. A través de las estructuras el sistema aprende de los sucesos y así, reproduce su identidad sistémica: "En esta propuesta de solución al problema de la doble contingencia (...) lo que se busca es resolver, fundamentalmente, es el problema de la capacidad de enlace de las operaciones de un sistema" (Luhmann 2002:330). 
Desde esta actualización de la teoría de los sistemas sociales, accedemos a una concepción de orden social sumamente dinámica que se muestra sensible a la contingencia. En el marco de la DC se constituyen estructuras que orientan al sistema y a su reproducción. No obstante, la reproducción del sistema no precisa de que las estructuras sean efectivas en la regulación de las acciones. A partir de las estructuras, el sistema puede observar a aquello que se distancia de las expectativas y actuar en consecuencia. El sistema no reproduce estructuras sino operaciones; las primeras resultan necesarias en tanto permiten condicionar la contingencia sin eliminarla. En esta dirección, todo acontecimiento que desafíe las selecciones sistémicas establecidas resulta plausible como aquello que no es esperado desde la perspectiva de la estructura. Es decir, como algo que recuerda al sistema acerca del carácter contingente de sus estructuras: "La formación de expectativas iguala un gran número de acontecimientos altamente heterogéneos al denominador común de la decepción de la expectativa, y designa así líneas de tratamiento" (Luhmann 1998:268).

El problema que detectamos en este movimiento es que aquello que trasciende la regulación de la estructura no puede ser explicado más que como un suceso casual; estimulado por la misma latencia de la DC. No es posible reconocer estructuras que los viabilicen. Son sucesos habilitados por la misma complejidad en la que opera el sistema. De esta manera, encontramos que con esta explicación del orden social se deja de lado la comprensión de estos fenómenos que, curiosamente, pretenden ser integrados. Desde nuestro punto de vista, esto tiene que ver con que el orden se concibe como regulación, condicionamiento o limitación de la contingencia, aunque ésta no pueda ser excluida. Aquí, la contingencia solo puede ser vista como la emergencia de lo que se presenta como inobservado por la estructura.

\section{El sistema social y el conflicto}

Atendiendo a la teoría de la DC, se hace reconocible el problema al que se enfrenta todo sistema social. Toda selección producida en el ámbito de la DC puede ser negada o rechazada por una selección posterior. Dada la contingencia de toda selección, esta alternativa siempre resulta factible al no existir ninguna seguridad ni certeza acerca de su estabilidad. A primera vista, esta posibilidad de rechazo podría poner en duda la continuidad del sistema. No obstante, Luhmann considera que el sistema se protege frente a esta amenaza por medio del conflicto que, como veremos, no es otra cosa que la comunicación de un rechazo o de una negación y la reestructuración que esto posibilita. El sistema puede continuar con sus operaciones por medio del conflicto. Luhmann define al conflicto como la comunicación de una contradicción, o también, como la contradicción de una comunicación. De esta manera, el conflicto remite a la existencia de dos comunicaciones que se presentan como contradictorias entre sí. Ante una expectativa, o a partir de una expectativa, se responde con una negación: "Los conflictos, por tanto, sirven, precisamente, para la continuación de la comunicación por medio de una de las posibilidades que mantiene abiertas: la utilización del no" (Luhmann 1998:350).

Para precisar el modo en que el conflicto permite la reproducción de las operaciones sistémicas, se hace preciso adentrarse en la función que Luhmann le asigna a las "contradicciones"; componente central del conflicto. Las contradicciones desempeñan funciones distintas según cómo sean contempladas. Si las abordamos desde aquel que las observa, evidentemente, la contradicción involucra una situación de indecisión, de inestabilidad, en tanto registra hechos que son mutuamente excluyentes. Por ejemplo, ahorrar y consumir. Resulta clara la imposibilidad de practicar estas dos acciones simultáneamente. En este caso, la contradicción "apaga" la observación. Ahora bien, para el sistema y para sus operaciones, la contradicción tiene la función de estimular una reacción; motiva a actuar: "en todos los sistemas autorreferenciales las contradicciones desempeñan una función doble: bloquean y desencadenan, frenan 
la observación que topa con la contradicción y se desprenden las operaciones de enlace relacionadas con las contradicciones que, precisamente por ello, son plenas de sentido" (Luhmann 1998:326).

Para Luhmann, las contradicciones permiten conservar la unidad del sistema cuando las expectativas se han mostrado inseguras. Podríamos decir que las contradicciones permiten la reproducción sistémica a partir de la desestabilización de las estructuras de los sistemas. Al poner en evidencia la incompatibilidad entre expectativas o entre una expectativa y una acción, dan cuenta de que las primeras se han tornado inadecuadas por alguna razón y que, debido a esto, se ha vuelto necesario actuar en consecuencia. Las expectativas ya no resultan útiles para enlazar o coordinar acciones y, por tanto, para lidiar con la DC. No obstante, esto no deja al sistema en una situación de total indeterminación ya que las contingencias se presentan en una relación de mutua exclusión. Ante esta situación, el sistema no elije entre las posibilidades incompatibles, sino que reacciona a la incompatibilidad misma. Luhmann destaca que no hay que entender a la desestabilización generada por la contradicción como disfuncional. En el contexto de la complejidad en la que operan los sistemas, dicha inestabilidad resulta fundamental para reaccionar a cambios en el entorno o a transformaciones internas. Las contradicciones brindan oportunidades para recrear la seguridad en las expectativas, para modificarlas o para crear otras nuevas.

En esta dirección, Luhmann no duda en sostener que el conflicto y la contradicción fungen como acontecimientos de inmunidad para el sistema. La inmunidad no tiene que ver con la corrección de las desviaciones o con la restitución del equilibrio propio de un estado anterior; no busca preservar las estructuras atacadas, sino que más bien busca la reproducción del sistema mismo gracias a las inestabilidades y no en contra de ellas. El sistema no se protege de la contradicción y el conflicto, sino que lo hace con su ayuda. En palabras de nuestro autor: "El sistema no se inmuniza contra la negación, sino con ayuda de la negación; no se protege contra los cambios, se protege contra la rigidez de los patrones de comportamiento habituales que han perdido vigencia respecto del entorno. El sistema de inmunidad no protege la estructura, sino la autopoiesis, la autorreproducción cerrada del sistema" (Luhmann 1998:335).

Esto involucra la posibilidad de integrar cambios y modificaciones que se consideren útiles. Todo conflicto, cualquier sea su origen, activa esta función inmunitaria; se constituye como una señal de alarma y de advertencia para el sistema. Lo que parecía coordinado ya no lo es, la complejidad determinada se transforma en indeterminada y ante la amenaza de que el sistema deje de existir, se insinúa que la acción debería seguir.

Los conflictos como comunicaciones de negaciones o rechazos y, así, como la manifestación de contradicciones al interior de los sistemas sociales, constituyen acontecimientos siempre posibles y hasta arbitrarios. Hecho que resulta compatible no solo con la teoría de la DC, sino también con la función de inmunidad que desempeñan los conflictos al interior de los sistemas. Si la posibilidad de rechazo estuviese clausurada, el sistema no tendría manera de producir acontecimientos de inmunidad. Adicionalmente, Luhmann se distancia de la tesis acerca de la existencia de contradicciones estructurales en el campo de lo social, es decir, de contradicciones que se presenten con independencia de los acontecimientos específicos que las constatan o actualizan. De ser así, los conflictos y las contradicciones no podrían desempeñar su función inmunitaria: "las contradicciones siempre presuponen una relación entre estructura y elemento (acontecimiento)" (Luhmann 1998:336).

Pese a que los conflictos son acontecimientos que se encuentran siempre a la mano en los sistemas sociales, Luhmann considera que existen ciertas condiciones que pueden probabilizarlos, es decir, que 
pueden activar el potencial de comunicación de una contradicción. A continuación, mencionamos, brevemente, algunas de las disposiciones hacia el conflicto que Luhmann reconoce en los sistemas sociales.

En primer lugar, el incremento de las posibilidades de comunicación estimula al conflicto. Por ejemplo, el desarrollo de los medios de difusión como la escritura, la imprenta, los medios masivos y, podríamos agregar, las redes sociales en la actualidad, brindan más oportunidades para el rechazo o la negación en tanto liberan a los intercambios de los controles propios de la interacción presencial. En segundo lugar, los medios de comunicación simbólicamente generalizados como la verdad, el poder, el dinero y el amor también desarrollan disposiciones para el conflicto. A la vez que estos medios cumplen la función de incrementar la probabilidad de aceptación de comunicaciones, también pueden ser útiles para advertir cuando se considere que no se cumplen con las condiciones que ellos imponen. Por ejemplo, cuando "las instituciones económicas de la propiedad y el dinero justifican el rechazo de exigencias exageradas de tributación" (Luhmann 1998:340); o cuando se genera un conflicto por una falta de compromiso en el caso del amor; o también, cuando en el medio de la verdad se rechaza a una teoría debido a que se considera falsa. En tercer lugar, Luhmann destaca al tiempo como un factor multiplicador de las contradicciones. En un mismo momento no se pueden ocupar dos espacios distintos. A la vez, las contradicciones se pueden resolver con ayuda del tiempo. Aunque no pueda estar simultáneamente en dos lugares al mismo tiempo, puedo estar primero en un lugar y luego en otro. Por esta razón, Luhmann considera que el tiempo mantiene una relación contradictoria con las contradicciones. Un ejemplo en relación con este tema lo pueden dar los problemas ecológicos y ambientales que muestran el modo en que el futuro y su previsión puede dar lugar a contradicciones en el presente. En cuarto lugar, ciertas experiencias sociales pueden ser disparadoras de conflictos. Por ejemplo, la competencia que presupone que las metas propias solo pueden alcanzarse a costa de las oportunidades de realización de las metas de los otros. Aunque la competencia en sí no implica una situación de contradicción, puede estimularla en caso de reconocerse a aquellos contra los cuales uno compite y así, entrar en conflicto con ellos: "La competencia únicamente refuerza la percepción de contradicción (...) cuando las opiniones o las intenciones de los demás se conciben como provocación de las propias" (Luhmann 1998:346). Por último, Luhmann advierte, en conexión con todas estas disposiciones, que su mayor presencia en la sociedad moderna es un indicio de que el aumento de la complejidad social ocasiona, por un lado, el aumento de las probabilidades de conflicto y, por el otro, la extensión del sistema de inmunidad, lo que implica una creciente sensibilidad hacia las perturbaciones y un aumento de inestabilidades.

De este modo, Luhmann muestra al conflicto como una estrategia fundamental para la reproducción de los sistemas sociales cuando las estructuras se presentan como incapaces de resolver adecuadamente el problema de la DC. A pesar de lo interesante de este tratamiento, encontramos que resulta parcialmente respondida la pregunta por aquello que habilita a la comunicación de una contradicción (al conflicto) y, por tanto, aquello que explica a estos acontecimientos y a las razones de su ocurrencia. Para Luhmann, las condiciones de posibilidad del conflicto se hayan, básicamente, en la contingencia de las estructuras que mantienen siempre abierta la alternativa del rechazo o la negación. Es esta contingencia la que parece explicar suficientemente la plausibilidad del conflicto. El rechazo puede servirse de estructuras, pero no es la estructura la que explica el rechazo. En este sentido, todo lo que podemos decir en torno al conflicto es que es un fenómeno siempre posible, aleatorio y, en todo caso, más o menos probable de acuerdo con el nivel de complejidad alcanzado por el sistema social. La teoría atribuye a la contingencia la explicación de los fenómenos conflictivos. Podríamos decir que el orden social no explica al conflicto, sino que su contingencia lo hace. Más allá de esto, Luhmann muestra que los conflictos devienen en un tipo de orden social específico al dar lugar a una resolución particular de la DC. Así, el conflicto no solo estimula a actuar, 
sino que también, bajo determinadas circunstancias, puede convertirse en una expectativa de comportamiento en el sistema social. Abordamos este planteo en el siguiente apartado.

\section{El conflicto como sistema social}

¿Qué es lo que sucede una vez que se ha comunicado una contradicción, expresado un rechazo o manifestado una negación? En este punto, Luhmann sostiene una hipótesis que tiene antecedentes en la teoría sociológica y que remite a la posibilidad de pensar al conflicto no como la disolución de lo social, sino, por el contrario, como un modo particular de vinculación, asociación o relación entre alteregos. Una de las tesis centrales del sociólogo alemán en torno al conflicto es que éstos configuran verdaderos sistemas sociales debido a que, a su manera, dan lugar al orden social a partir de una resolución particular al problema de la contingencia social. Una vez que se ha presentado un conflicto, se hace posible la orientación de los comportamientos a partir de considerar al otro como contrario o enemigo. De este modo, pese a que el origen del conflicto pueda ser arbitrario y contingente, una vez ocurrido queda "regido por la DC" (Pignuoli 2013:33), es decir, se presenta como una forma específica de reducción de complejidad en el marco de la constelación alter-ego. Así, no son solo los acuerdos y el consenso los que viabilizan el orden, sino que también los conflictos lo posibilitan.

Al poner en evidencia la insuficiencia de las estructuras para regular el orden social, el conflicto posibilita el enlace de las acciones más allá de que existan dudas acerca de las expectativas que se deben seguir. El conflicto ocupa el lugar de las estructuras en el sistema; las partes ya no se orientan como lo hacen normalmente por expectativas específicas de comportamiento, sino por medio de una expectativa más amplia y general que remite al patrón de conflicto: no hago lo que quieres si no haces lo que quiero. Luhmann observa que este esquema implica, a pesar de todo, una forma de resolver el problema de la DC. El sistema social se orienta por la expectativa de que todo lo que hará el otro me perjudicará o no me beneficiará: "lo que daña a alter es considerado por ego (en principio, limitadamente, luego de manera general) como su propia ventaja, cosa que vale para alter" (Luhmann 1998:351).

Contrariamente a lo que se podría suponer, Luhmann observa que estos sistemas son fuertemente integradores al tender a subordinar toda acción a la lucha. Integración no quiere decir aquí algo funcional o positivo para el grupo, sino principalmente, reducción de grados de libertad. Esto significa que toda acción desarrollada en el contexto de la rivalidad aparece como un aspecto de la rivalidad misma. En relación con esto, los conflictos vuelven altamente interdependientes a las partes, debido a que las motivaciones para la acción se encuentran atadas a los comportamientos del contrincante.

Los conflictos logran algo que en vano se ha buscado mediante llamados a la lealtad: un efecto de unión muy considerable en relación con la interpenetración y la estructura. Esto no es solo válido para la solidaridad en el interior en los partidos en disputa, sino, y justamente también, para la rivalidad misma. Quien pierde a su enemigo, siente un vacío peculiar, pues le faltan motivos de acción a los que él mismo se había obligado.

Otra característica de los sistemas de conflicto es su carácter expansivo y así, potencialmente peligroso para el sistema social al interior del cual se forman. Cuando el conflicto comienza a generalizarse como expectativa, cualquier conducta puede ser vista como asociada al conflicto. Es decir, el conflicto es expansivo en tanto puede dar lugar a una dinámica de apropiación de todo tipo de elementos y recursos del sistema para reproducirse. Todo es interpretado en términos de contradicción con los objetivos propios. Todo lo que sucede en el sistema social es reducido estructuralmente a la bilateralidad del 
conflicto. Por esta razón, Luhmann habla de los conflictos como sistemas "parásitos" que tienden a absorber toda la energía y los recursos de otros sistemas. De este modo, el conflicto no es destructivo por sí mismo, sino que lo puede ser en relación con el sistema en el cual apareció. Podríamos decir, el conflicto sirve como un mecanismo de inmunidad, pero también, si no se contiene, puede dar lugar a la desaparición del sistema. Por ejemplo, cuando alguien se cansa de discutir y decide abandonar la interacción; aquello que podía ayudar al sistema se transforma en su sentencia de muerte: "es apropiada la metáfora de la existencia parasitaria de conflictos; aunque, por lo general, se distingue del parasitismo por el hecho de que no persigue simbiosis, dado que tiende a la absorción del sistema anfitrión por parte del conflicto" (Luhmann 1998:351).

La equivalencia entre conflicto y sistema social nos permite llevar nuestro argumento un paso adelante. Fundamentalmente, destacamos que esta tesis no aporta a la comprensión de las razones del conflicto (que echábamos en falta en la definición del conflicto exhibida en el apartado anterior). La pregunta que orienta a Luhmann no es cómo es posible el conflicto, sino cómo este es manejado en el sistema social. Solo pudimos reconocer que el conflicto, una vez que aparece, no trae aparejada una desorganización del sistema, debido a que no deja a la acción social en una situación de total indeterminación. El conflicto desempeña una función estructurante en el sistema. En este caso, es observable contundentemente el vínculo entre orden social y conflicto. Ahora bien, esta tesis nos permite confirmar que el problema del orden social es visto como un problema de control, hecho que dificulta la comprensión de las razones por las cuales emergen o se manifiestan los conflictos. Nótese que el conflicto puede ser observado como un tipo de orden social en tanto se configura como una estructura que logra regular y condicionar las acciones del sistema. El conflicto representa un equivalente funcional con respecto a las estructuras o expectativas normales de los sistemas, y en eso radica la plausibilidad de su conformación como sistema. En este caso, nuevamente, no es el orden social el que viabiliza al conflicto. Más bien, es el conflicto el que, a su modo, viabiliza el orden social. Podríamos decir, el conflicto no representa, en primera instancia, una amenaza para el orden social, en tanto otorga condicionamientos específicos para continuar con las acciones del sistema. El conflicto escapa a la regulación de la estructura, no obstante, como tal permite lidiar con la DC.

\section{El conflicto y la sociedad}

Si bien Luhmann destaca en reiteradas instancias que el conflicto es un hecho cotidiano, frecuente, masivo, banal, aleatorio y hasta arbitrario, en el marco de su teoría de los sistemas sociales desea explicar la razón por la cual algunos de ellos se vuelven más significativos, generan efectos más amplios y desarrollan una "carrera social importante" (Luhmann 1998:353). Mientras que la gran mayoría de los conflictos sociales se desactivan rápidamente, se debilitan, se solucionan o dejan de despertar interés; otros parecen estabilizarse y alcanzar una amplia relevancia societal, es decir, producen "consecuencias importantes durante un largo periodo con efectos en gran escala social" (Luhmann 1998:353). En este punto, nuestro autor cuestiona (en sintonía con la conceptualización que venimos reconstruyendo) la idea de que los conflictos relevantes serían solo aquellos estructuralmente determinados como, por ejemplo, los de clase o los de dominación. En primer lugar, porque esto implicaría negar la multiplicidad de circunstancias que dan lugar a los conflictos y, en segundo lugar, porque limitaría el potencial del sistema de inmunidad al restringir la variedad de conflictos significativos. En definitiva, a Luhmann no le interesa distinguir un tipo especial de conflicto especialmente relevante, sino que desea comprender las condiciones que posibilitan que ciertos conflictos adquieran una relevancia social más amplia que otros.

Para atender a este problema, Luhmann propone distinguir entre interacción y sociedad. En este marco, la inquietud queda reformulada por el modo en que un conflicto puede trascender la interacción y 
proyectarse hacia la sociedad. En esta dirección, la sociedad debe otorgar criterios que permitan reconocer la relevancia social de determinados conflictos y que, de este modo, favorezcan su extensión más allá de las situaciones interactivas. De lo contrario, el gran volumen de conflictos permanecería desapercibido sin ningún tipo de relevancia y, por tanto, sin poder ser utilizados y procesados por el sistema inmunológico de la sociedad. En esta dirección, Luhmann habla de condicionamientos de conflictos: "Para que una sociedad reproduzca su sistema de inmunidad, debe ofrecer suficientes posibilidades de conflictos aún abiertas. Puesto que el sistema debe ser móvil y rápidamente motivante, esto no puede ocurrir por la mera prescripción del inicio del conflicto, sino por medio del condicionamiento de las perspectivas de reproducción del conflicto que comenzaron por causas fútiles. Por lo tanto, la sistematización de los acontecimientos de inmunidad no puede explicarse en el nivel de los sistemas de conflicto particulares interactivos; solo es posible como un agregado social de los muchos conflictos posibles" (Luhmann 1998:355).

Algunas de las referencias externas que pueden condicionar los conflictos y así, seleccionarlos para que se extiendan más allá de los contextos de interacción, pueden ser otros sistemas sociales como la política, la economía, el derecho o la moral. Por ejemplo, cuando la temática de un conflicto parece tener relevancia política o cuando los contendientes en un conflicto desean sostener que sus posturas son públicamente correctas. También, cuando la resolución de una disputa requiere de la intervención de un tribunal que decida en torno a la misma. Adicionalmente, las organizaciones también pueden tener la capacidad de seleccionar conflictos y extenderlos. Aquí podríamos pensar en los sindicatos, las asociaciones profesionales o también ciertas organizaciones no gubernamentales. De esta manera, la sociedad constituye un sistema de inmunidad que, mediante el condicionamiento, hace posible que no se excluyan aquellos conflictos que puedan ser relevantes en sus consecuencias para la reproducción del sistema social.

Sin embargo, esto no responde la pregunta por la carrera exitosa de los conflictos. ¿Cómo se seleccionan aquellos conflictos que cobrarán importancia? En principio, lo visto hasta aquí solo permite reconocer a aquello que da lugar a que las disputas trasciendan el carácter circunstancial de una interacción. Pero, qué es lo que lleva a que un conflicto adquiera importancia en la sociedad. Sobre este punto, Luhmann se limita a plantear dos procedimientos o mecanismos que servirían para ello. Por un lado, el derecho, que mediante el reforzamiento de posiciones políticas y económicas (de poder y de propiedad), puede legitimar al conflicto. En las sociedades tradicionales estas dimensiones se encontraban menos diferenciadas, por lo que era de esperar que la disposición para el conflicto fuera menor. En la sociedad moderna, en cambio, la diferenciación funcional entre derecho, política y economía ha permitido que el derecho logre una mayor independencia y, por tanto, otorgue más oportunidades para la manifestación del conflicto. Por el otro, se encuentran los movimientos sociales, los cuales permiten que los conflictos se extiendan en el tiempo a partir del logro de adhesiones y compromisos individuales para con determinados objetivos, que pese a ser poco claros o específicos, permiten ubicar adversarios, reunir disposiciones de conflicto y, de este modo, direccionar a la acción común. Ambas formas de extensión y desarrollo de conflictos (el derecho y los movimientos sociales) contribuyen al sistema de inmunidad de la sociedad. Por esta razón, Luhmann entiende que no resulta del todo adecuado considerarlos como procedimientos opuestos: unos a favor de la estructura, los otros en contra de ellas.

Para el sociólogo alemán, en ambas instancias de lo que se trata es, justamente, de reproducir la sociedad cuando las estructuras se muestran insuficientes. En palabras del autor: "En el marco de esta formación selectiva de la contradicción y el conflicto, el reforzamiento de las posiciones negativas por medio del derecho y de la manifestación de agitaciones, de la crítica y de protestas en forma de movimientos 
sociales, ha adquirido una significación complementaria. En la usual argumentación sociohistórica, ambas soluciones se contraponen: el complejo político económico derivado del capitalismo moderno y la globalidad de los movimientos sociales que esto provoca. Teóricamente sería más productivo distinguir entre estructura de expectativa y sistema de inmunidad. Así, por lo menos, se evidenciaría que la sociedad moderna, comparada con los procesos históricos desestabiliza las estructuras y aumenta considerablemente el potencial de negación. Será entonces menos importante saber si él no se articula más desde las posiciones del poder, del derecho, o desde el contexto de los movimientos sociales" (Luhmann 1998:362).

Del mismo modo que lo hemos mostrado en los apartados anteriores, encontramos que la integración del conflicto en la teoría de la sociedad de Luhmann tampoco da respuesta a la tensión que venimos advirtiendo. Específicamente, la teoría de la sociedad no presenta una explicación en torno a las razones que motivan la emergencia de los conflictos. El sociólogo alemán reafirma que los conflictos son fenómenos aleatorios que surgen permanentemente en las interacciones y de circunstancias de lo más variadas. Consecuentemente con los puntos anteriores, para la emergencia del conflicto basta con que se comunique un No. Alternativa que el sistema siempre tiene a la mano. Partiendo de esta premisa, lo que le interesa a Luhmann es mostrar los mecanismos que permiten seleccionar aquellos conflictos que podrían tener una relevancia mayor para la sociedad. En conexión con esto se destaca la existencia de mecanismos que organizan esos conflictos en patrones significativos. Por otra parte, el derecho y los movimientos sociales, son dos mecanismos claves para el reforzamiento e institucionalización de los conflictos. A partir de estos planteos, Luhmann argumenta, de un modo similar al que venimos mostrando, que el conflicto es compatible con la sociedad (con el orden social), de hecho, es necesario en tanto ésta debe mantener abiertas suficientes posibilidades de conflictos para desarrollar su sistema de inmunidad. La sociedad no se protege contra el conflicto, sino gracias a él. Dicho esto, la teoría de la sociedad no se ocupa de comprender sus razones, sino de atender al modo en que, como sistema social, puede condicionar, regular, manejar, reproducir y extender los conflictos. La pregunta que orienta la indagación no es por qué emergen los conflictos, sino cómo la sociedad los canaliza y los utiliza para su supervivencia.

\section{Consideraciones finales}

Al comenzar este trabajo, marcábamos que uno de los aportes fundamentales, y ampliamente reconocido de la teoría de los sistemas sociales de Niklas Luhmann, es su sensibilidad para con la complejidad y la contingencia del mundo. Premisas que se muestran productivas para la integración de aquellos fenómenos que tradicionalmente fueron considerados ajenos al orden social como, por ejemplo, el conflicto, la variación, la creatividad, el cambio, la evolución o la desviación. En este espacio, nos propusimos analizar específicamente el modo en el que el conflicto es integrado en la teoría general de los sistemas sociales. Es verdad que para tradiciones sociológicas (como la marxista) el conflicto, definitivamente, no se presenta como un fenómeno residual en la explicación del orden social. De hecho, lo contrario es cierto: el conflicto es constitutivo del orden. Sin embargo, Luhmann desea evitar esta subordinación del orden al conflicto. Si este fuere el caso, la teoría de sistemas sociales debería dejar de lado otras formas de actualización del orden como, por ejemplo, el consenso, el cual solo podría ser comprendido como un derivado de una situación conflictiva fundamental. Para la teoría sistémica, el orden social no puede quedar reducido al conflicto. Propuesta que limitaría la riqueza del mundo social al negar la contingencia de esta forma de actualización del orden. Adicionalmente, se obturaría la posibilidad de comprender la función específica que desempeñan los conflictos al interior del sistema social, al instaurarse como su única y exclusiva condición de posibilidad. Además, frente a las teorías del orden social que ubican al conflicto como un emergente del desajuste entre los intereses individuales y la sociedad, el teórico alemán sostiene que el 
conflicto no debe concebirse como un acontecimiento meramente disruptivo. También debe ser reconocido como un hecho siempre actualizable en los sistemas sociales, que se vuelve fundamental cuando las estructuras se experimentan como poco adecuadas para la coordinación de las acciones $u$ operaciones del sistema.

En esta dirección, el objetivo de Luhmann es proponer una teoría del sistema social lo suficientemente abstracta y general como para poder integrar al conflicto como una forma de orden particular. A pesar de lo novedoso de este tratamiento, nuestra pretensión en este texto fue la de exhibir una potencial limitación. Fundamentalmente, observamos que el planteo luhmanniano presenta dificultades para dar cuenta cabalmente de las razones que efectivamente motivan o explican la emergencia de un conflicto. En principio, solo podríamos dar cuenta de su carácter ineludible, de su mayor o menor frecuencia y de sus consecuencias para el sistema. Debido a esto, sostuvimos la hipótesis de que la integración teórica propuesta resulta parcial. El orden social es compatible con el conflicto, de hecho, el conflicto es un tipo de orden social, no obstante, no es el orden quien puede explicarlo o definir las razones de su ocurrencia o manifestación.

En nuestro análisis encontramos que la posible causa de esta dificultad radica en la asimilación de una concepción regulativa de orden social que no se haya suficientemente problematizada. La inevitabilidad de la complejidad y la contingencia como dimensiones irreductibles de la realidad social representan problemas con los que debe lidiar el orden para sobrevivir. Para esto, se configuran sistemas que por medio de estructuras reducen la complejidad y limitan la contingencia, sin obturarlas. Por este motivo, para Luhmann, el control o la regulación que puede ejercer el sistema no se subordina a la estabilidad o a la reproducción de las estructuras. De hecho, el sistema contempla la posibilidad de que las estructuras sean defraudadas. Así, el sistema opera en una dialéctica entre estructura y contingencia. El sistema dispone de estructuras contingentes que permiten lidiar con la contingencia de las operaciones del sistema. Nótese que, si bien la contingencia de los acontecimientos es asumida, resulta difícil para la teoría dar cuenta o explicar a aquellos acontecimientos y/o selecciones que implican algún tipo de decepción con respecto a las estructuras. La teoría de sistemas no niega esos acontecimientos, sin embargo, tampoco los puede explicar más que como emergentes de la complejidad y la contingencia de las estructuras sistémicas.

Desde nuestra perspectiva, las dificultades para dar cuenta de las razones del conflicto se hayan en esta manera de comprender al orden como condicionamiento, limitación, regulación o control de la contingencia por medio de estructuras. En este sentido, la teoría del orden social solo puede explicar al conflicto como algo que escapa al control de la estructura (aunque, no necesariamente, al control del sistema), que pone en evidencia su incapacidad de coordinación y que, por consiguiente, no se encuentra habilitado o motivado por ella. Es la complejidad misma, en la que se asienta el sistema, la que impulsa a los conflictos. El orden se basa en la capacidad del sistema para manejarlos y procesarlos. Esto permite comprender por qué razón Luhmann se concentra en explicar las consecuencias, las funciones y las lógicas sociales que pone en marcha el conflicto, más que en las condiciones que lo viabilizan; sobre estas últimas basta con afirmar que son una posibilidad (más o menos probable), siempre abierta para los sistemas sociales que operan en el medio del sentido y a través de la comunicación.

Para enmendar esta situación consideramos que se perfilan dos alternativas. Por un lado, se podría retomar una opción de la que Luhmann pretende distanciarse. Esta consistiría en sostener que los conflictos sociales se encuentran estructuralmente determinados. Esto significaría asumir que en los sistemas sociales no habría otra cosa más que conflicto. Los conflictos se encontrarían predefinidos; y en 
todo caso, en estado de latencia. De hecho, no se precisaría de que se manifiesten para poder ser constatados por el teórico social. En este caso, mantendríamos una imagen de orden social como control, pero a diferencia de Luhmann, se subordinaría el control a la reproducción de la estructura, algo que, como vimos, también es cuestionado por la teoría de los sistemas.

Ahora bien, si deseamos mantener el supuesto de la contingencia y la complejidad en la comprensión del conflicto y, a la vez, mantener la capacidad explicativa de la teoría, consideramos que una segunda alternativa podría radicar en dejar de concebir al orden social como control de la acción. Es decir, de concebir a la contingencia como aquello que se escapa a la regulación de la estructura, pese a ser asimilada por el sistema. Para esto, deberemos entender a la función de la estructura no como el condicionamiento de las acciones sociales, sino como su misma condición de posibilidad. En un trabajo previo argumentamos que concebir de esta manera a las estructuras del orden social, no debería llevarnos a excluir la posibilidad de identificar otras que, efectivamente, tuviesen la función de controlar la contingencia. Desde este lugar, sin negar la contingencia de los acontecimientos conflictivos, podremos comprender mejor su emergencia al relacionarlos con determinadas estructuras y no, meramente, con la contingencia de las mismas. Así, será factible visualizar la procedencia de las fuerzas del conflicto. Pensar al conflicto como aquello que trasciende a la regulación estructural tiene sentido, solamente, si entendemos al orden social como control.

\section{Bibliografía}

Fuchs, S. 2000. Dos cambios paradigmáticos en la teoría sociológica sistémica: Niklas Luhmann. Sociológica 15(43): 205-215. http://www.sociologicamexico.azc.uam.mx/index.php/Sociologica/article/view/486

Luhmann, N. 1998. Sistemas sociales. Lineamientos para una teoría general. Madrid: Anthropos.

Luhmann, N. 2002. Introducción a la teoría de los sistemas. Madrid: Anthropos.

Pignuoli, S. 2013. Doble contingencia y orden social desde la teoría de los sistemas de Niklas Luhmann. Sociológica 28(78): 7-40.

http://www.sociologicamexico.azc.uam.mx/index.php/Sociologica/article/view/50

Recibido el 13 Ago 2017

Aceptado el 24 Nov 2017 